\title{
ON DEDEKIND'S CRITERION AND MONOGENICITY OVER DEDEKIND RINGS
}

\author{
M. E. CHARKANI and O. LAHLOU
}

Received 29 November 2002

\begin{abstract}
We give a practical criterion characterizing the monogenicity of the integral closure of a Dedekind ring $R$, based on results on the resultant $\operatorname{Res}\left(P, P_{i}\right)$ of the minimal polynomial $P$ of a primitive integral element and of its irreducible factors $P_{i}$ modulo prime ideals of $R$. We obtain a generalization and an improvement of the Dedekind criterion (Cohen, 1996) and we give some applications in the case where $R$ is a discrete valuation ring or the ring of integers of a number field, generalizing some well-known classical results.
\end{abstract}

Mathematics Subject Classification: 11Y40, 13A18, 13F30.

1. Introduction. Let $K$ be an algebraic number field and let $O_{K}$ be its ring of integers. If $O_{K}=\mathbb{Z}[\theta]$ for some number $\theta$ in $O_{K}$, we say that $O_{K}$ has a power basis or $O_{K}$ is monogenic. The question of the existence of a power basis was originally examined by Dedekind [5]. Several number theorists were interested in and attracted by this problem (see [7, 8, 9]) and noticed the advantages of working with monogenic number fields. Indeed, for a monogenic number field $K$, in addition to the ease of discriminant computations, the factorization of a prime $p$ in $K / \mathbb{Q}$ can be found most easily (see [4, Theorem 4.8.13, page 199]). The main result of this paper is Theorem 2.5 which characterizes the monogenicity of the integral closure of a Dedekind ring. More precisely, let $R$ be a Dedekind domain, $K$ its quotient field, $L$ a finite separable extension of degree $n$ of $K, \alpha$ a primitive element of $L$ integral over $K, P(X)=\operatorname{Irrd}(\alpha, K)$, $m$ a maximal ideal of $R$, and $O_{L}$ the integral closure of $R$ in $L$. Assume that $\bar{P}(X)=\prod_{i=1}^{r} \bar{P}_{i}^{e_{i}}(X)$ in $(R / m)[X]$ with $e_{i} \geq 2$, and let $P_{i}(X) \in R[X]$ be a monic lifting of $\bar{P}_{i}(X)$ for $1 \leq i \leq r$. Then we prove that $O_{L}=R[\alpha]$ if and only if, for every maximal ideal $m$ of $R$ and $i \in\{1, \ldots, r\}, v_{m}\left(\operatorname{Res}\left(P_{i}, P\right)\right)=\operatorname{deg}\left(P_{i}\right)$, where $v_{m}$ is the $m$-adic discrete valuation associated to $m$. This leads to a necessary and sufficient condition for a simple extension $R[\alpha]$ of a Dedekind ring $R$ to be Dedekind. At the end, we give two illustrations of this criterion. In the second example, we give the converse which was not known yet.

2. Monogenicity over a Dedekind ring. Throughout this paper $R$ is an integral domain, $K$ its quotient field, $L$ is a finite separable extension of degree $n$ of $K, \alpha$ is a primitive element of $L$ integral over $R, P(X)=\operatorname{Irrd}(\alpha, K), m$ is 
a maximal ideal of $R$, and $O_{L}$ is the integral closure of $R$ in $L$. Let $f$ and $g$ be two polynomials over $R$; the resultant of $f$ and $g$ will be denoted by $\operatorname{Res}(f, g)$ (see [11]).

DEFINITION 2.1. If $O_{L}=R[\theta]$ for some number $\theta \in O_{L}$, then $O_{L}$ has a power basis or $O_{L}$ is monogenic.

Proposition 2.2. Let $R$ be an integrally closed ring and let $\alpha$ be an integral element over $R$. Then $(R[\alpha])_{p}=R_{p}[\alpha]$ for every prime ideal $p$ of $R$. In particular, $O_{L}=R[\alpha]$ if and only if $R_{p}[\alpha]$ is integrally closed for every prime ideal $p$ of $R$ if and only if $R[\alpha]$ is integrally closed.

Proof. We obtain the result from the isomorphism $R[\alpha] \simeq R[X] /\langle P(X)\rangle$, the properties of an integrally closed ring and its integral closure, and the properties of a multiplicative closed subset of a ring $R$, notably, $S^{-1}(R[X])=$ $\left(S^{-1} R\right)[X]$ (see [1]).

DEFINITION 2.3. Let $R$ be a discrete valuation ring (DVR), $p=\pi R$ its maximal ideal, and $\alpha$ an integral element over $R$. Let $P$ be the minimal polynomial of $\alpha$, and $\bar{P}(X)=\prod_{i=1}^{r} \bar{P}_{i}^{e_{i}}(X)$ the decomposition of $\bar{P}$ into irreducible factors in $(R / p)[X]$. Set

$$
\begin{aligned}
f(X) & =\prod_{i=1}^{r} P_{i}(X) \in R[X], \\
h(X) & =\prod_{i=1}^{r} P_{i}^{e_{i}-1}(X) \in R[X], \\
T(X) & =\frac{P(X)-\prod_{i=1}^{r} P_{i}^{e_{i}}(X)}{\pi} \in R[X],
\end{aligned}
$$

where $P_{i}(X) \in R[X]$ is a monic lifting of $\bar{P}_{i}(X)$, for $1 \leq i \leq r$. We will say that $R[\alpha]$ is $p$-maximal if $(\bar{f}, \bar{T}, \bar{h})=1$ in $(R / p)[X]$ (where $(\cdot, \cdot)$ denotes the greatest common divisor (gcd)). If $R$ is a Dedekind ring and $p$ is a prime ideal of $R$, then we say that $R[\alpha]$ is $p$-maximal if $R_{p}[\alpha]$ is $p R_{p}$-maximal.

REMARKS 2.4. (1) If $\pi$ is uniramified in $R[\alpha]$, that is, $e_{i}=1$ for all $\mathrm{i}$, then $\bar{h}=\overline{1}$ and therefore $R[\alpha]$ is $p$-maximal.

(2) Let $\pi$ be ramified in $R[\alpha]$, that is, there is at least one $i$ such that $e_{i} \geq 2$. Let $S=\left\{i \in\{1, \ldots, r\} \mid e_{i} \geq 2\right\}$ and $f_{1}(X)=\prod_{i \in S} P_{i}(X) \in R[X]$. Then $\left(\bar{f}_{1}, \bar{T}\right)=(\bar{T}, \bar{f}, \bar{h})$ in $(R / p)[X]$ since $\bar{f}_{1}=(\bar{f}, \bar{h})$. In particular, if every $e_{i} \geq 2$, then $(\bar{f}, \bar{T})=(\bar{T}, \bar{f}, \bar{h})$, because $\bar{f}$ divides $\bar{h}$ in this case.

(3) Definition 2.3 is independent of the choice of the monic lifting of the $\bar{P}_{i}$. More precisely, let

$\bar{P}(X)=\prod_{i=1}^{r} \bar{P}_{i}^{e_{i}}(X)=\prod_{i=1}^{r} \bar{Q}_{i}^{e_{i}}(X) \quad$ with $\bar{P}_{i}(X)=\bar{Q}_{i}(X)$ for $1 \leq i \leq r$ in $(R / p)[X]$. 
Set

$$
\begin{gathered}
g(X)=\prod_{i=1}^{r} Q_{i}(X) \in R[X], \quad k(X)=\prod_{i=1}^{r} Q_{i}^{e_{i}-1}(X) \in R[X] \\
U(X)=\pi^{-1}\left(P(X)-\prod_{i=1}^{r} Q_{i}^{e_{i}}(X)\right) \in R[X] .
\end{gathered}
$$

Then $(\bar{f}, \bar{T}, \bar{h})=1$ in $(R / p)[X]$ if and only if $(\bar{g}, \bar{U}, \bar{k})=1$ in $(R / p)[X]$. Indeed, we may assume that $R$ is a DVR and $p=\pi R$. Let $V_{1}=(g-f) / \pi$ and $V_{2}=$ $(k-h) / \pi$. Then $\pi T=\pi U+g k-f h$. Replacing $g$ by $\pi V_{1}+f$ and $k$ by $\pi V_{2}+h$, we find that $\bar{T}=\bar{U}+\bar{V}_{1} \bar{h}+\bar{V}_{2} \bar{f}$ and therefore $(\bar{T}, \bar{f}, \bar{h})=(\bar{U}, \bar{f}, \bar{h})=(\bar{U}, \bar{g}, \bar{k})$ since $\bar{f}=\bar{g}$ and $\bar{h}=\bar{k}$.

THEOREM 2.5. Let $R$ be a Dedekind ring. Let $P$ be the minimal polynomial of $\alpha$, and assume that for every prime ideal $p$ of $R$, the decomposition of $\bar{P}$ into irreducible factors in $(R / p)[X]$ verifies:

$$
\bar{P}(X)=\prod_{i=1}^{r} \bar{P}_{i}^{e_{i}}(X) \in(R / p)[X]
$$

with $e_{i} \geq 2$ for $i=1, \ldots, r$ and $P_{i}(X) \in R[X]$ be a monic lifting of the irreducible factor $\bar{P}_{i}$ for $i=1, \ldots, r$. Then $O_{L}=R[\alpha]$ if only if $v_{p}\left(\operatorname{Res}\left(P_{i}, P\right)\right)=\operatorname{deg}\left(P_{i}\right)$ for every prime ideal $p$ of $R$ and for every $i=1, \ldots, r$, where $v_{p}$ is the $p$-adic discrete valuation associated to $p$.

For the proof we need the following two lemmas.

LEMMA 2.6. Let $p=u R+v R$ be a maximal ideal of a commutative ring $R$. Then $p R_{p}=v R_{p}$ if and only if there exist $a, b \in R$ such that $u=a u^{2}+b v$.

Proof. If $p R_{p}=v R_{p}$, then there exist $s \in R$ and $t \in R-p$ such that $t u=v s$. Since $p$ is maximal in $R$, so there exists $t^{\prime} \in R$ such that $t t^{\prime}-1 \in p$. Hence $u-u t t^{\prime}=u-v s t^{\prime} \in p^{2}$ and there exist $a, b \in R$ such that $u=a u^{2}+b v$. Conversely, $u^{2} R+v R \subseteq v R+p^{2} \subseteq p$. If there exist $a, b \in R$ such that $u=$ $a u^{2}+b v$, then $p=u^{2} R+v R$ and therefore $v R+p^{2}=p$. Localizing at $p$ and applying Nakayama's lemma, we find that $p R_{p}=v R_{p}$.

LEMMA 2.7. Let $R$ be a commutative integral domain, let $K$ be its quotient field, and consider $P, g, h, T \in R[X]$. If $g$ is monic and $P=g h+\pi T$, then $\operatorname{Res}(g, P)=\pi^{\operatorname{deg}(g)} \operatorname{Res}(g, T)$. In particular, if $m=\pi R$ is a maximal ideal of $R$ and if $\bar{P}(X)=\prod_{i=1}^{r} \bar{P}_{i}^{e_{i}}(X)$ is the decomposition of $\bar{P}$ into irreducible factors in $(R / m)[X]$, with $P_{i}(X) \in R[X]$ a monic lifting of $\bar{P}_{i}(X)$ for $1 \leq i \leq r$, and $T(X)=\pi^{-1}\left(P(X)-\prod_{i=1}^{r} P_{i}^{e_{i}}(X)\right) \in R[X]$, then

$$
\operatorname{Res}\left(P_{i}, P\right)=\pi^{\operatorname{deg}\left(P_{i}\right)} \operatorname{Res}\left(P_{i}, T\right)
$$


and $\left(\bar{P}_{i}, \bar{T}\right)=1$ in $(R / m)[X]$ if and only if

$$
\operatorname{Res}\left(P_{i}, T\right)=\frac{\operatorname{Res}\left(P_{i}, P\right)}{\pi^{\operatorname{deg}\left(P_{i}\right)}} \in R-m
$$

Proof. Let $x_{1}, \ldots, x_{m}$ be the roots of $g$ in the algebraic closure $\bar{K}$ of $K$. It is then easy to see (see [11]) that $\operatorname{Res}(g, P)=\prod_{i=1}^{m} P\left(x_{i}\right)=\pi^{\operatorname{deg}(g)} \operatorname{Res}(g, T)$ because $P\left(x_{i}\right)=\pi T\left(x_{i}\right)$. The second result follows from $\operatorname{Res}\left(\bar{P}_{i}, \bar{P}\right)=\overline{\operatorname{Res}}\left(P_{i}, P\right)$ and [2, Corollary 2, page 73].

Proof of TheOrem 2.5. By Proposition 2.2, we may assume that $R$ is a DVR. Let $p$ be a prime ideal of $R$ and $\left(O_{L}\right)_{(p)}$ the integral closure of $R_{p}$ in $L$. Let $\bar{P}(X)=\Pi_{i=1}^{r} \bar{P}_{i}^{e_{i}}(X)$ in $\left(R_{p} / p R_{p}\right)[X]$ with $e_{i} \geq 2$ and $P_{i}(X) \in R_{p}[X]$ a monic lifting of $\bar{P}_{i}(X)$ for $1 \leq i \leq r$. Let

$$
T(X)=\frac{P(X)-\Pi_{i=1}^{r} P_{i}^{e_{i}}(X)}{\pi} \in R_{p}[X]
$$

with $\pi R_{p}=p R_{p}$.

(a) We prove that if $\left(\bar{P}_{i}, \bar{T}\right)=1$ in $\left(R_{p} / p R_{p}\right)[X]$ for every $i=1, \ldots, r$, then $\left(O_{L}\right)_{(p)}=R_{p}[\alpha]=A$. Indeed, $\bar{P}(X)=\Pi_{i=1}^{r} \bar{P}_{i}^{e_{i}}(X)$ in $\left(R_{p} / p R_{p}\right)[X]$ and $R_{p}$ is a local ring, so by [14, Lemma 4 , page 29] (see also [3]) the ideals $\mathscr{B}_{i}=\pi A+P_{i}(\alpha) A$ $(i=1, \ldots, r)$ are the only maximal ideals of $A$, so $A$ is integrally closed if and only if $\mathscr{A}_{\mathscr{B}_{i}}$ is integrally closed for every $i=1, \ldots, r$. More generally, we prove that every $\mathscr{A}_{\mathscr{B}_{i}}$ is a DVR. Since $R_{p}$ is Noetherian, so $R_{p}[\alpha] \simeq R_{p}[X] /\langle P(X)\rangle$ is Noetherian, hence $\mathscr{A}_{\mathscr{B}_{i}}$ is Noetherian since $\mathscr{A}_{\mathscr{B}_{i}}$ is a local integral domain with maximal ideal $\mathscr{B}_{i} \mathscr{A}_{\mathscr{B}_{i}}$. It remains to show that $\mathscr{B}_{i} \mathscr{A}_{\mathscr{B}_{i}}$ is principal. Indeed, $\left(\bar{P}_{i}, \bar{T}\right)=1$ in $\left(R_{p} / p R_{p}\right)[X]$, hence there exist polynomials $U_{1}, U_{2}, U_{3} \in$ $R_{p}[X]$ such that $1=U_{1}(X) P_{i}(X)+U_{2}(X) T(X)+\pi U_{3}(X)$. Now $P(\alpha)=0=$ $\Pi_{j=1}^{r} P_{j}^{e_{j}}(\alpha)+\pi T(\alpha)$, hence $\Pi_{j=1}^{r} P_{j}^{e_{j}}(\alpha)=-\pi T(\alpha)$, so

$$
\begin{aligned}
\pi & =\pi U_{1}(\alpha) P_{i}(\alpha)+\pi^{2} U_{3}(\alpha)-\Pi_{j=1}^{r} P_{j}^{e_{j}}(\alpha) U_{2}(\alpha) \\
& =\pi^{2} U_{3}(\alpha)+P_{i}(\alpha) U_{4}(\alpha)
\end{aligned}
$$

with $U_{4}=\pi U_{1}-P_{i}^{e_{i}-1}\left(\prod_{j=1, j \neq i}^{r} P_{j}^{e_{j}}\right) U_{2} \in R_{p}[X]$. It follows from Lemma 2.6 that $\mathscr{B}_{i} \mathscr{A}_{\mathscr{B}_{i}}=P_{i}(\alpha) \mathscr{A}_{\mathscr{B}_{i}}$, in other words, $\mathscr{B}_{i} \mathscr{A}_{\mathscr{B}_{i}}$ is principal. We conclude that $\mathscr{A}_{\mathscr{B}_{i}}$ is a DVR and therefore an integrally closed ring, and $\left(O_{L}\right)_{(p)}=R_{p}[\alpha]$.

(b) We will now prove that $\left(\bar{P}_{i}, \bar{T}\right)=1$ in $\left(R_{p} / p R_{p}\right)[X]$ for every $i=1, \ldots, r$ if $\left(O_{L}\right)_{(p)}=R_{p}[\alpha]$. We first show that the ring $\mathscr{A}_{\mathscr{B}_{i}}$ is a DVR, for every $i$. Indeed, $R_{p}$ is a Dedekind ring and $L$ is a finite extension of $K$, and it follows from [10, Theorem 6.1, page 23] that $\left(O_{L}\right)_{(p)}=R_{p}[\alpha]=A$ is a Dedekind ring, so $\mathscr{A}_{\mathscr{B}_{i}}$ is a DVR. Let us show next that $T(\alpha)$ is a unit in every $\mathscr{A}_{\mathscr{B}_{i}}$. Indeed, $\mathscr{A}_{\mathscr{B}_{i}}$ is a DVR and so its maximal ideal $\mathscr{S}_{i} \mathscr{A}_{\mathscr{B}_{i}}=\pi \mathscr{A}_{\mathscr{B}_{i}}+P_{i}(\alpha) \mathscr{A}_{\mathscr{B}_{i}}$ is principal. Let $\lambda \in \mathscr{A}_{\mathscr{B}_{i}}$ be a generator of $\mathscr{B}_{i} \mathscr{A}_{\mathscr{B}_{i}}$. Then there exist $u, v \in \mathscr{A}_{\mathscr{B}_{i}}$ such that $\lambda=\pi u+$ $P_{i}(\alpha) v \in \mathscr{B}_{i} \mathscr{A}_{\mathscr{B}_{i}}-\left(\mathscr{B}_{i} \mathscr{A}_{\mathscr{B}_{i}}\right)^{2}$. Now $R_{p}$ is a DVR, $P=\operatorname{Irrd}\left(\alpha, R_{p}\right), \bar{P}=\Pi_{j=1}^{r} \bar{P}_{j}^{e_{j}}$ 
in $\left(R_{p} / \pi R_{p}\right)[X], \pi R_{p} \in \operatorname{Spec} R_{p}$, and $\left(O_{L}\right)_{(p)}=R_{p}[\alpha]=A$ is the integral closure of $R_{p}$ in $L=K(\alpha)$ with $K=\operatorname{Fr}\left(R_{p}\right)$, and we find that $\pi A=\Pi_{j=1}^{r} \mathscr{B}_{j}^{e_{j}}$. Hence $\pi \in \mathscr{B}_{i}{ }^{2}$ because $e_{i} \geq 2$. Now $\lambda \notin\left(\mathscr{B}_{i} \mathscr{A}_{\mathscr{B}_{i}}\right)^{2}$, hence $P_{i}(\alpha) \notin\left(\mathscr{B}_{i} \mathscr{A}_{\mathscr{B}_{i}}\right)^{2}$, because $\lambda=u \pi+P_{i}(\alpha) v$. It then follows that $P_{i}(\alpha)$ is a generator of $\mathscr{S}_{i} \mathscr{A}_{\mathscr{B}_{i}}=P_{i}(\alpha) \mathscr{A}_{\mathscr{B}_{i}}$ since $\pi \mathscr{A}_{\mathscr{B}_{i}}=\left(\mathscr{B}_{i} \mathscr{A}_{\mathscr{B}_{i}}\right)^{e_{i}}=P_{i}^{e_{i}}(\alpha) \mathscr{A}_{\mathscr{B}_{i}}$, and $\pi=P_{i} e_{i}(\alpha) \epsilon_{1}$ with $\epsilon_{1} \in U\left(\mathscr{A}_{\mathscr{B}_{i}}\right)$. We now show that $P_{j}(\alpha) \in U\left(\mathscr{A}_{\mathscr{B}_{i}}\right)$ for every $j \neq i$. Indeed, if $P_{j}(\alpha) \in \mathscr{B}_{i} \mathscr{A}_{\mathscr{B}_{i}}$, then there exists $a_{i} \in \mathscr{B}_{i}$ and $b_{i} \in A-\mathscr{B}_{i}$ such that $P_{j}(\alpha)=a_{i} / b_{i}$. Then $a_{i}=P_{j}(\alpha) b_{i} \in \mathscr{B}_{i}$. Now, $\mathscr{B}_{i}$ is a prime ideal of A, hence $P_{j}(\alpha) \in \mathscr{B}_{i}$. As $\mathscr{B}_{j}=$ $\pi A+P_{j}(\alpha) A$, so $\mathscr{B}_{j} \subseteq \mathscr{B}_{i}$. The ideal $\mathscr{B}_{j}$ is a maximal ideal of $\mathrm{A}$, so $\mathscr{B}_{i}=\mathscr{B}_{j}$. This is impossible because the $\mathscr{B}_{i}$ are distinct, and it follows that $P_{j}(\alpha) \in U\left(\mathscr{A}_{\mathscr{B}_{i}}\right)$ for every $j \neq i$. Thus there exists $\epsilon_{2} \in U\left(\mathscr{A}_{\mathscr{B}_{i}}\right)$ such that $\prod_{j=1, j \neq i}^{r} P_{j}^{e_{j}}(\alpha)=\epsilon_{2}$. Since $\prod_{j=1}^{r} P_{j}^{e_{j}}(\alpha)=-\pi T(\alpha), \pi=P_{i}^{e_{i}}(\alpha) \epsilon_{1}$, and $\prod_{j=1, j \neq i}^{r} P_{j}^{e_{j}}(\alpha)=\epsilon_{2}$, then $T(\alpha)=-\epsilon_{2} \epsilon_{1}^{-1} \in U\left(\mathscr{A}_{\mathscr{B}_{i}}\right)$. So $T(\alpha) \in U\left(\mathscr{A}_{\mathscr{B}_{i}}\right)$ for every $i$, and $T(\alpha) \in U(A)$; otherwise, Krull's theorem implies the existence of a maximal ideal $\mathscr{B}_{i}$ of A such that $T(\alpha) \in \mathscr{B}_{i}$, and $T(\alpha) \in \mathscr{B}_{i} \mathscr{A}_{\mathscr{B}_{i}}=\mathscr{A}_{\mathscr{B}_{i}}-U\left(\mathscr{A}_{\mathscr{B}_{i}}\right)$, which is impossible. We conclude that $T(\alpha)$ is a unit in $R_{p}[\alpha]$, and, by [2, Corollary 1, page 73], there exist $U_{1}, V_{1} \in R_{p}[X]$ such that $1=U_{1}(X) P(X)+V_{1}(X) T(X)$. Consequently $\overline{1}=$ $\bar{U}_{1}(X) \bar{P}(X)+\bar{V}_{1}(X) \bar{T}(X)$ in $\left(R_{p} / \pi R_{p}\right)[X]$, which is principal. Hence $(\bar{P}, \bar{T})=$ 1 in $\left(R_{p} / \pi R_{p}\right)[X]$ since $\bar{P}=\prod_{i=1}^{r} \bar{P}_{i}^{e_{i}}$ in $\left(R_{p} / \pi R_{p}\right)[X]$ then $\left(\bar{P}_{i}, \bar{T}\right)=1$ in $\left(R_{p} / \pi R_{p}\right)[X]$ for every $i$. Our result now follows from Proposition 2.2 and Lemma 2.7.

REMARKS 2.8. (1) Let $\pi$ be ramified in $R[\alpha], S=\left\{i \in\{1, \ldots, r\} \mid e_{i} \geq 2\right\}$, and $f_{1}(X)=\prod_{i \in S} P_{i}(X) \in R[X]$. It follows from Lemma 2.7 that the following statements are equivalent:

(i) $\left(\bar{f}_{1}, \bar{T}\right)=1$ in $(R / p)[X]$;

(ii) $v_{p}\left(\operatorname{Res}\left(f_{1}, P\right)\right)=\operatorname{deg}\left(f_{1}\right)$;

(iii) for every $i \in S$, we have $v_{p}\left(\operatorname{Res}\left(P_{i}, P\right)\right)=\operatorname{deg}\left(P_{i}\right)$, where $v_{p}$ is the $p$-adic discrete valuation associated to $p$.

(2) It follows from the above equivalence and Remark 2.4(2) and (3) that the condition in Theorem 2.5 is independent of the choice of the monic lifting of $\bar{P}_{i}$. More precisely, if $e_{i} \geq 2$ for every $i$, and if we take another monic lifting $Q_{i}$ of $\bar{P}_{i}$, then $v_{p}\left(\operatorname{Res}\left(P_{i}, P\right)\right)=\operatorname{deg}\left(P_{i}\right)$ for all $i=1, \ldots, r$ if and only if $v_{p}\left(\operatorname{Res}\left(Q_{i}, P\right)\right)=$ $\operatorname{deg}\left(Q_{i}\right)$ for all $i=1, \ldots, r$.

(3) Theorem 2.5 states that, under the assumption that $e_{i} \geq 2$ for every $i$, $O_{L}=R[\alpha]$ if and only if $R[\alpha]$ is $p$-maximal for every prime ideal $p$ of $R$.

COROLLARY 2.9. Under the assumptions of Theorem 2.5, if $O_{L}=R[\alpha]$, then, for every prime ideal $p$ of $R, R_{p}[\alpha]$ is principal and $\mathscr{S}_{i}=P_{i}(\alpha) R_{p}[\alpha]$ for every $i$.

PROoF. Indeed, a Dedekind ring having only a finite number of prime ideals is principal. To prove the second statement, take $x \in A$ such that $\mathscr{B}_{i}=x A$. Then $\mathscr{P}_{i} \mathscr{A}_{\mathscr{B}_{i}}=x \mathscr{A}_{\mathscr{B}_{i}}=P_{i}(\alpha) \mathscr{A}_{\mathscr{B}_{i}}$, hence $P_{i}(\alpha)=x \varepsilon$ with $\varepsilon \in U\left(\mathscr{A}_{\mathscr{B}_{i}}\right)$. Then $\varepsilon \in U(A)$, so $\mathscr{B}_{i}=P_{i}(\alpha) A$. 
DEFINITION 2.10. Let $R$ be a DVR with maximal ideal $m=\pi R$, with $f, g \in$ $R[X]$ monic polynomials. Then $f$ is called an Eisenstein polynomial relative to $g$ if there exists $T \in R[X]$ and an integer $e \geq 1$ such that $f=g^{e}+\pi T$ and $(\bar{g}, \bar{T})=1$ in $(R / \pi R)[X]$.

REMARK 2.11. As in the classical Eisenstein's criterion, we have a criterion for the irreducibility of an Eisenstein polynomial relative to $g$, called the Schönemann criterion, see [12, page 273]; if $f=g^{e}+\pi T$ is an Eisenstein polynomial relative to $g$ such that $\bar{g} \in(R / m)[X]$ is irreducible and $\operatorname{deg}(T)<e \operatorname{deg}(g)$, then $f$ is irreducible in $K[X]$.

COROLLARY 2.12. Let $R$ be a DVR with maximal ideal $m=\pi R$. If $\bar{P}=\bar{g}^{e}$ in $(R / m)[X]$ withe $\geq 2$, then $O_{L}=R[\alpha]$ if and only if $P$ is an Eisenstein polynomial relative to $g$.

Proof. We obtain the result using Theorem 2.5, Definition 2.10, and Lemma 2.7 .

REMARK 2.13. Corollary 2.12 generalizes [14, Propositions 15 and 17]; it integrates the two results in one statement and provides the converse.

3. Monogenicity over the ring of integers. Let $K=\mathbb{Q}(\alpha)$ be a number field of degree $n, P(X) \in \mathbb{Z}[X]$ a minimal polynomial of $\alpha, O_{K}$ the ring of integers of $K$, and $p$ a prime number.

Proposition 3.1. Let $K=\mathbb{Q}(\alpha)$ be a number field and $P$ the minimal polynomial of $\alpha$. Then $O_{K}=\mathbb{Z}[\alpha]$ if and only if for every prime number $p$ such that $p^{2}$ divides $\operatorname{Disc}(P)$, the prime number $p$ does not divide $\operatorname{Ind}(\alpha)$.

Proof. We obtain the result from the fact that $O_{K}=\mathbb{Z}[\alpha]$ if and only if $\operatorname{Ind}(\alpha)=1$, and $\operatorname{Disc}(P)=(\operatorname{Ind}(\alpha))^{2} d_{K}$ (see [6], [4, page 166]).

Proposition 3.2. Let $\bar{P}(X)=\prod_{i=1}^{r} \bar{P}_{i}^{e_{i}}(X)$ be the factorization of $P(X)$ modulo $p$ in $\mathbb{F}_{p}[X]$, and put $f(X)=\prod_{i=1}^{r} P_{i}(X)$ with $P_{i}(X) \in \mathbb{Z}[X]$ a monic lifting of $\bar{P}_{i}(X)$ and $e_{i} \geq 2$ for all $i$. Let $h(X) \in \mathbb{Z}[X]$ be a monic lifting of $\bar{P}(X) / \bar{f}(X)$ and $T(X)=(f(X) h(X)-P(X)) / p \in \mathbb{Z}[X]$. Then the following statements are equivalent:

(i) $p$ does not divide $\operatorname{Ind}(\alpha)=\left[O_{K}: \mathbb{Z}[\alpha]\right]$;

(ii) $(\bar{f}, \bar{T})=1$ in $\mathbb{F}_{p}[X]$;

(iii) $v_{p}(\operatorname{Res}(f, P))=\operatorname{deg}(f)$;

(iv) $v_{p}\left(\operatorname{Res}\left(P_{i}, P\right)\right)=\operatorname{deg}\left(P_{i}\right)$, for every $i \in\{1, \ldots, r\}$.

Proof. (i) $\Leftrightarrow$ (ii). Let $\left(O_{K}\right)_{(p)}$ be the integral closure of $\mathbb{Z}_{(p)}$ in $K$. We first show that $p$ does not divide $\operatorname{Ind}(\alpha)$ if and only if $\left(O_{K}\right)_{(p)}=\mathbb{Z}_{(p)}[\alpha]$. By the finiteness theorem [13, page 48], $\left(O_{K}\right)_{(p)}=\oplus_{i=0}^{n-1} \mathbb{Z}_{(p)} x_{i}$, and, because $\mathbb{Z}_{(p)}$ is principal, $\alpha^{i}=\sum_{j=0}^{n-1} a_{i j} x_{j}$ with $a_{i j} \in \mathbb{Z}_{(p)}$, and therefore $\left[\left(O_{K}\right)_{(p)}: \mathbb{Z}_{(p)}[\alpha]\right]=\left|\operatorname{det}\left(a_{i j}\right)\right|$. 
On the other hand, Ind $(\alpha)=\left[O_{K}: \mathbb{Z}[\alpha]\right]=\left[\left(O_{K}\right)_{(p)}:(\mathbb{Z}[\alpha])_{(p)}\right]=\left[\left(O_{K}\right)_{(p)}\right.$ : $\left.\mathbb{Z}_{(p)}[\alpha]\right]$, hence $\left(O_{K}\right)_{(p)}=\mathbb{Z}_{(p)}[\alpha]$ if and only if $p$ does not divide $\operatorname{Ind}(\alpha)$ if and only if $\operatorname{Ind}(\alpha) \in \cup\left(\mathbb{Z}_{(p)}\right)=\mathbb{Z}_{(p)}-p \mathbb{Z}_{(p)}$. Hence by the proof of Theorem 2.5, $p$ does not divide Ind $(\alpha)$ if and only if $\left(\bar{P}_{i}, \bar{T}\right)=1$ in $\mathbb{F}_{p}[X]$ for every $i=1,2, \ldots, r$ (in other words, if and only if $(\bar{f}, \bar{T})=1$ in $\left.\mathbb{F}_{p}[X]\right)$.

(ii) $\Leftrightarrow$ (iii). By [2, Corollary 2, page 73$],(\bar{f}, \bar{T})=1$ in $\mathbb{F}_{p}[X]$ if and only if $\operatorname{Res}(\bar{f}, \bar{T})=\overline{\operatorname{Res}}(f, T) \neq \overline{0}$ in $\mathbb{F}_{p}$ if and only if $\operatorname{Res}(f, T) \in \mathbb{Z}-p \mathbb{Z}$. On the other hand,

$$
\operatorname{Res}(f, T)=\frac{(-1)^{\operatorname{deg}(f)}}{p^{\operatorname{deg}(f)}} \operatorname{Res}(f, P)
$$

(ii) $\Leftrightarrow$ (iv). We have $(\bar{f}, \bar{T})=1$ in $\mathbb{F}_{p}[X]$ if and only if $\operatorname{Res}(f, T) \in \mathbb{Z}-p \mathbb{Z}$. On the other hand, $\operatorname{Res}(f, T)=\prod_{i=1}^{r} \operatorname{Res}\left(P_{i}, T\right)$ and

$$
\operatorname{Res}\left(P_{i}, T\right)=\frac{(-1)^{\operatorname{deg}\left(P_{i}\right)}}{p^{\operatorname{deg}\left(P_{i}\right)}} \operatorname{Res}\left(P_{i}, P\right) .
$$

THEOREM 3.3. Let $K=\mathbb{Q}(\alpha)$ be a number field of degree $n, P(X) \in \mathbb{Z}[X]$ a monic minimal polynomial of $\alpha$, and $O_{K}$ the ring of integers of $K$. Assume $\bar{P}(X)=\prod_{i=1}^{r} \bar{P}_{i}^{e_{i}}(X)$ in $\mathbb{F}_{p}[X]$, for every prime number $p$ such that $p^{2}$ divides $\operatorname{Disc}(P)$, with $P_{i}(X) \in \mathbb{Z}[X]$ a monic lifting of $\bar{P}_{i}(X)$ and $e_{i} \geq 2$ for $1 \leq i \leq r$. Then $O_{K}=\mathbb{Z}[\alpha]$ if and only if for every prime number $p$, such that $p^{2}$ divides $\operatorname{Disc}(P), v_{p}\left(\operatorname{Res}\left(P_{i}, P\right)\right)=\operatorname{deg}\left(P_{i}\right)$ for $1 \leq i \leq r$.

Proof. It suffices to apply Propositions 3.1 and 3.2, and Theorem 2.5.

REMARK 3.4. Proposition 3.2 provides a complement to the Dedekind criterion (see [4, page 305]). Indeed, in $\mathbb{F}_{p}[X]$, we have $(\bar{f}, \bar{T})=(\bar{f}, \bar{T}, \bar{h})$ since all $e_{i} \geq 2$.

We finish this section giving other conditions equivalent to $p$ not being a divisor of $\operatorname{Ind}(\alpha)$.

Proposition 3.5. The following statements are equivalent:

(i) $p$ does not divide $\operatorname{Ind}(\alpha)=\left[O_{K}: \mathbb{Z}[\alpha]\right]$;

(ii) $\mathbb{Z}[\alpha]+p O_{K}=O_{K}$;

(iii) $\mathbb{Z}[\alpha] \cap p O_{K}=p \mathbb{Z}[\alpha]$.

Proof. (ii) $\Leftrightarrow$ (iii). Consider the following map of $\mathbb{F}_{p}$-vector spaces:

$$
j: \mathbb{Z}[\alpha] / p \mathbb{Z}[\alpha] \longrightarrow O_{K} / p O_{K}, \quad j(x+p \mathbb{Z}[\alpha])=x+p O_{K} .
$$

As $O_{K}$ and $\mathbb{Z}[\alpha]$ are two free groups of the same rank $n, \mathbb{Z}[\alpha] / p \mathbb{Z}[\alpha]$ and $O_{K} / p O_{K}$ are two $\mathbb{F}_{p}$-vector spaces of the same dimension $n$ and injectivity of $j$ is equivalent to surjectivity of $j$. Moreover, $j$ is one-to-one if and only if $\mathbb{Z}[\alpha] \cap p O_{k}=p \mathbb{Z}[\alpha]$ and $j$ is onto if and only if $\mathbb{Z}[\alpha]+p O_{K}=O_{K}$. 
(i) $\Leftrightarrow$ (iii). If $p$ does not divide $\operatorname{Ind}(\alpha)$ and $p \mathbb{Z}[\alpha] \subset \mathbb{Z}[\alpha] \cap p O_{K}$, then there exists $x \in O_{K}$ such that $x \notin \mathbb{Z}[\alpha]$ and $p x \in \mathbb{Z}[\alpha]$, so the order of the subgroup generated by $x+\mathbb{Z}[\alpha]$ of the finite group $O_{K} / \mathbb{Z}[\alpha]$ is equal to $p$, and, by Lagrange's theorem, $p$ divides $\operatorname{Ind}(\alpha)$, which is the order of the group $O_{K} / \mathbb{Z}[\alpha]$, and this is impossible.

Conversely, assume that $\mathbb{Z}[\alpha] \cap p O_{K}=p \mathbb{Z}[\alpha]$ and $p$ divides Ind $(\alpha)$. Cauchy's theorem implies that there exists an element of order $p$ in $O_{K} / \mathbb{Z}[\alpha]$; in other words, there exists $x \in O_{K}$ such that $x \notin \mathbb{Z}[\alpha]$ and $p x \in \mathbb{Z}[\alpha]$. Then $p x \in$ $\mathbb{Z}[\alpha] \cap p O_{K}=p \mathbb{Z}[\alpha]$, hence $x \in \mathbb{Z}[\alpha]$, which is impossible.

\section{Applications}

\subsection{Monogenicity of cyclotomic fields}

Proposition 4.1. Let $n \geq 3$ be an integer, $\xi_{n}$ a primitive $n$th root of unity, $K=\mathbb{Q}\left(\xi_{n}\right)$, and $\phi_{n}(X)$ the nth cyclotomic polynomial over $\mathbb{Q}$. Then $O_{K}=\mathbb{Z}\left[\xi_{n}\right]$.

Proof. We know from [15] that

$$
\begin{aligned}
\phi_{n}(X) & =\prod_{\substack{1 \leq i \leq n \\
i \wedge n=1}}\left(X-\xi_{n}^{i}\right)=\operatorname{Irrd}\left(\xi_{n}, \mathbb{Q}\right) \\
\operatorname{Disc}\left(\phi_{n}\right) & =(-1)^{\varphi(n) / 2} \frac{n^{\varphi(n)}}{\prod_{p \mid n} p^{\varphi(n) /(p-1)}}=(-1)^{\varphi(n) / 2} \prod_{i=1}^{s} p_{i}^{\varphi(n)\left(r_{i}-1 /\left(p_{i}-1\right)\right)},
\end{aligned}
$$

where $\varphi(n)$ is the Euler $\varphi$-function and

$$
n=\prod_{i=1}^{s} p_{i}^{r_{i}}=p_{i}^{r_{i}} m_{i} \quad \text { with } m_{i}=\prod_{j=1, j \neq i}^{s} p_{j}^{r_{j}} .
$$

Let $q$ be a prime number such that $q^{2}$ divides $\operatorname{Disc}\left(\phi_{n}\right)$. Then there exists $i \in$ $\{1, \ldots, s\}$ such that $q=p_{i}$. We have $\bar{\phi}_{n}(X)=\left(\bar{\phi}_{m_{i}}(X)\right)^{\varphi\left(p_{i}^{r_{i}}\right)}\left(\bmod p_{i}\right)$, where $\varphi\left(p_{i}^{r_{i}}\right) \geq 2$, and

$$
\operatorname{Res}\left(\phi_{m_{i}}, \phi_{n}\right)=(-1)^{\varphi\left(m_{i}\right) \varphi(n)} \operatorname{Res}\left(\phi_{n}, \phi_{m_{i}}\right)=\operatorname{Res}\left(\phi_{n}, \phi_{m_{i}}\right)=p_{i}^{\varphi\left(m_{i}\right)},
$$

and we obtain that $v_{p_{i}}\left(\operatorname{Res}\left(\phi_{n}, \phi_{m_{i}}\right)\right)=\operatorname{deg}\left(\phi_{m_{i}}(X)\right)$.

Now the result follows immediately from Theorem 3.3 and Proposition 3.2.

4.2. Monogenicity of the field $K=\mathbb{Q}(\alpha)$, with $\alpha$ a root of $P(X)=X^{p}-a$

Proposition 4.2. Let $\alpha$ be a root of the irreducible polynomial $P(X)=X^{p}-$ $a$, where $a$ is a squarefree integer and $p$ is a prime number. 
(i) If $p$ divides $a$, then $O_{K}=\mathbb{Z}[\alpha]$ if and only if $a$ is squarefree.

(ii) If $p$ does not divide $a$, then $O_{K}=\mathbb{Z}[\alpha]$ if and only if $a$ is squarefree and $v_{p}\left(a^{p-1}-1\right)=1$.

Proof. We have $P(X)=X^{p}-a=\operatorname{Irrd}(\alpha, \mathbb{Q})$ and

$$
\operatorname{Disc}(P)=(-1)^{p((p-1) / 2)} N_{K / \mathbb{Q}}\left(P^{\prime}(\alpha)\right)=(-1)^{\left(3 p^{2}-p-2\right) / 2} p(a p)^{p-1}
$$

If $p$ is odd, the only prime numbers $q$ such that $q^{2}$ divides $\operatorname{Disc}(P)$ are $p$ and the prime divisors of $a$. If $p=2$, then 2 is the only prime number $q$ such that $q^{2}$ divides $\operatorname{Disc}(P)$.

Let $q$ be a prime number such that $q^{2}$ divides $\operatorname{Disc}(P)$. We have two cases:

(1) if $q$ does not divide $a$, then $\bar{P}(X)=\overline{\mathcal{g}(X)}^{p}$ in $\mathbb{F}_{p}[X]$, with $g(X)=X-a$, and then $\operatorname{Res}(g, P)=P(a)=a^{p}-a$;

(2) if $q$ divides $a$, then $\bar{P}(X)=\overline{\mathcal{g}(X)}^{p}$ in $\mathbb{F}_{q}[X]$, with $g(X)=X$ and then $\operatorname{Res}(g, P)=P(0)=-a$.

In both cases, the result is deduced from Theorem 3.3.

\section{REFERENCES}

[1] M. F. Atiyah and I. G. Macdonald, Introduction to Commutative Algebra, AddisonWesley, Massachusetts, 1969.

[2] N. Bourbaki, Algèbre, Masson, Paris, 1981.

[3] M. Charkani, Structure multiplicative des idéaux d'une extension primitive d'un anneau intégralement clos, in preparation.

[4] H. Cohen, A Course in Computational Algebraic Number Theory, Graduate Texts in Mathematics, vol. 138, Springer-Verlag, Berlin, 1996.

[5] R. Dedekind, Über den Zussamenhang zwischen der Theorie der Ideals und der Theorie der hoheren Cyclotimy index, Abh. Akad. Wiss. Göttingen Math.Phys. Kl. 23 (1878), 1-23 (German).

[6] A. Fröhlich and M. J. Taylor, Algebraic Number Theory, Cambridge Studies in Advanced Mathematics, vol. 27, Cambridge University Press, Cambridge, 1993.

[7] M.-N. Gras, Sur les corps cubiques cycliques dont l'anneau des entiers est monogène, C. R. Acad. Sci. Paris Sér. A 278 (1974), 59-62 (French).

[8] _ Z Z-bases d'entiers $1, \theta, \theta^{2}, \theta^{3}$ dans les extensions cycliques de degré 4 de $\mathbf{Q}$ [Z-bases of integers $1, \theta, \theta^{2}, \theta^{3}$ in cyclic extensions of degree 4 of Q], Number Theory, Publ. Math. Fac. Sci. Besançon, Université de FrancheComté, Besançon, 1981, pp. 1-14 (French).

[9] _ Non monogénéité de l'anneau des entiers des extensions cycliques de $\mathbf{Q}$ de degré premier $l \geq 5$ [Nonmonogeneity of the ring of integers of cyclic extensions of $\mathbf{Q}$ of prime degree $l \geq 5$ ], J. Number Theory 23 (1986), no. 3, 347-353 (French).

[10] G. J. Janusz, Algebraic Number Fields, Pure and Applied Mathematics, Academic Press, New York, 1973.

[11] S. Lang, Algebra, 2nd ed., Addison-Wesley, Massachusetts, 1984.

[12] P. Ribenboim, Théorie des Valuations, Les presses de l'université de Montréal, Montréal, 1964 (French).

[13] P. Samuel, Théorie Algébrique des Nombres, Hermann, Paris, 1971 (French). 
[14] J.-P. Serre, Corps Locaux, Publications de l'Université de Nancago, Hermann, Paris, 1968 (French).

[15] L. C. Washington, Introduction to Cyclotomic Fields, Graduate Texts in Mathematics, vol. 83, Springer-Verlag, New York, 1982.

M. E. Charkani: Department of Mathematics, Faculty of Sciences Dhar-Mahraz, University of Sidi Mohammed Ben Abdellah, BP 1796, Fes, Morocco

E-mail address: mcharkani@excite.com

O. Lahlou: Department of Mathematics, Faculty of Sciences Dhar-Mahraz, University of Sidi Mohammed Ben Abdellah, BP 1796, Fes, Morocco

E-mail address: 1. ouafae@caramai1.com 


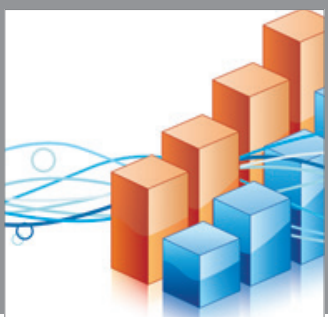

Advances in

Operations Research

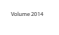

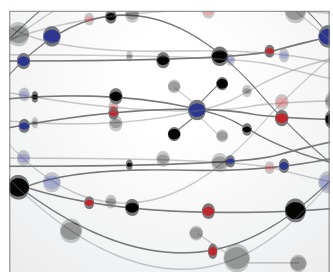

\section{The Scientific} World Journal
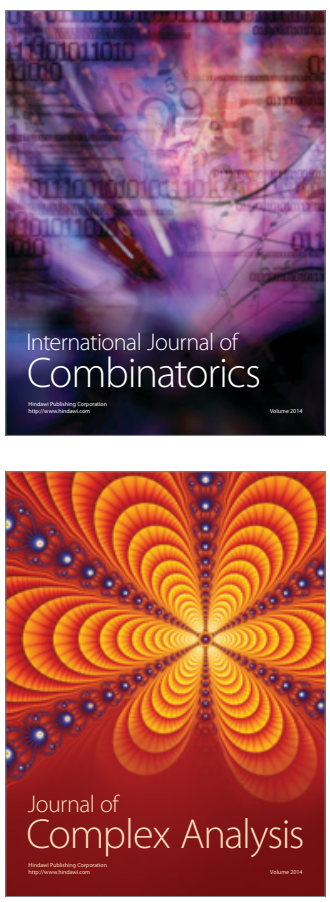

International Journal of

Mathematics and

Mathematical

Sciences
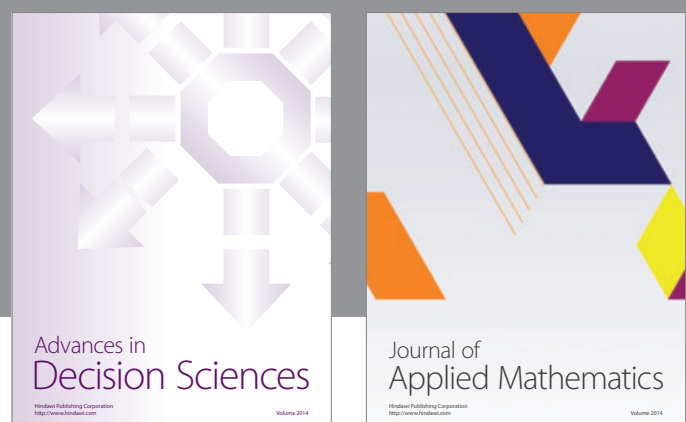

Journal of

Applied Mathematics
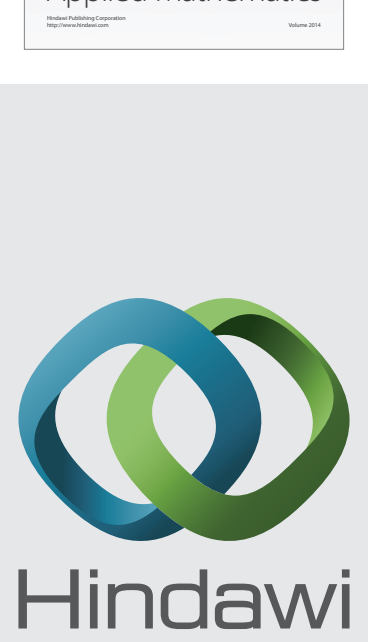

Submit your manuscripts at http://www.hindawi.com
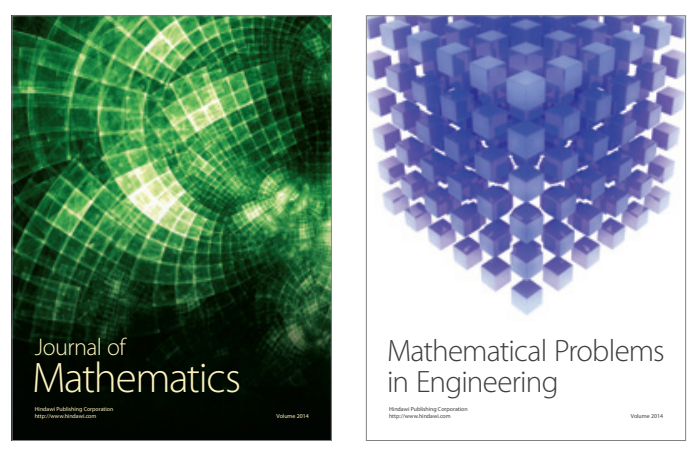

Mathematical Problems in Engineering
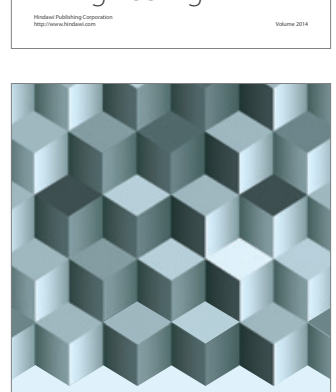

Journal of

Function Spaces
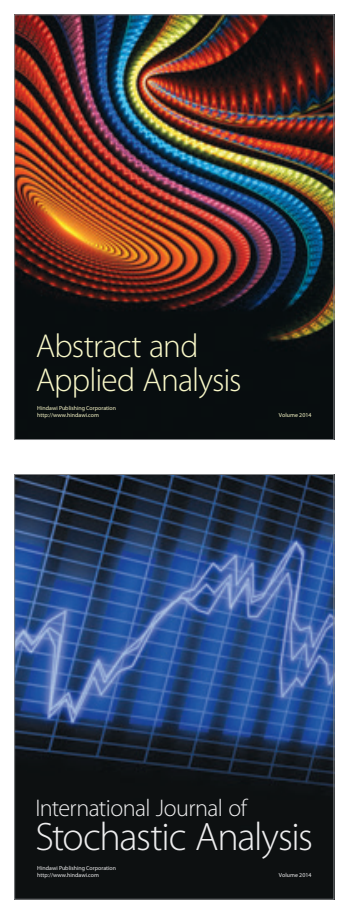

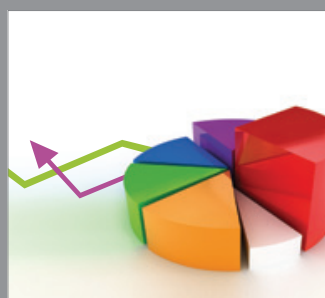

ournal of

Probability and Statistics

Promensencen
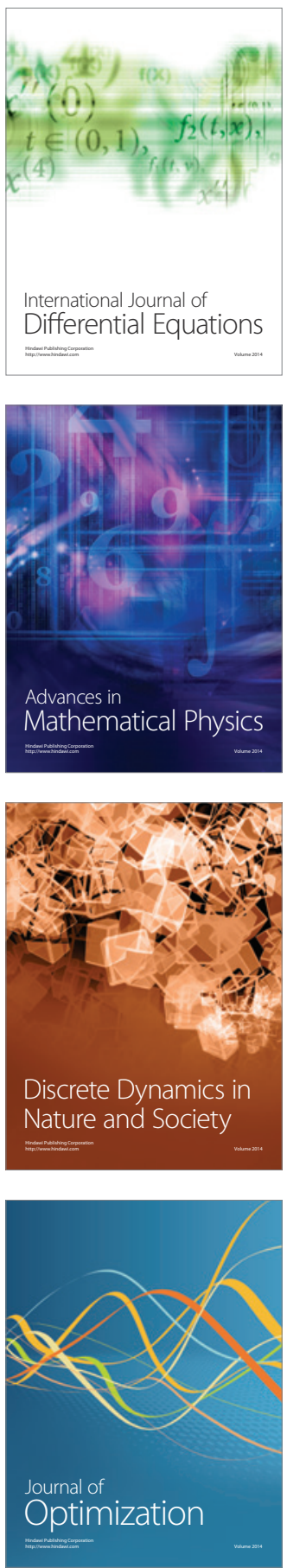